FETAL AND NEONATAL EDITION

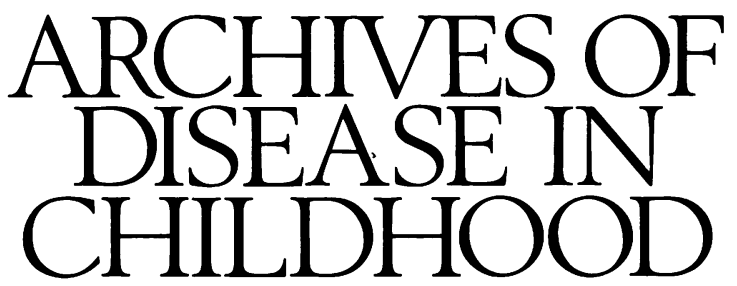

The fournal of the British Paediatric Association

\title{
Do we need an Apgar score?
}

In the 38 years that have elapsed since Virginia Apgar described a scoring system for the assessment of the condition of infants at birth, the Apgar score has achieved legendary status as the 'gold standard' outcome measure for countless studies. In light of modern perinatal practice, it is important to assess the value of the score - does it still measure what it originally intended to? Is the score being used appropriately? Can we improve on it?

\section{Recording the Apgar score}

The scoring system was designed to be made by an independent observer at one minute after delivery as an indicator of immediate newborn condition, in order to guide appropriate delivery room management. ${ }^{1}$ To this a further score at five minutes is added, and it is common practice to record the score every five minutes or so while resuscitation continues. The Apgar score is therefore a shorthand method of recording the clinical condition of a newborn infant, relying on five signs - heart rate, respiration, muscle tone, reflex irritability, and colour - that should normally be assessed when deciding to proceed with a resuscitation.

In most situations the scoring is incorrect because the staff who have carried out the resuscitation generally make the assessment. In practice the record is made retrospectively and can only indicate what the attendants recall. This may be some considerable time after delivery should the child be intubated and ventilated to the intensive care nursery, the attending doctor being asked to complete the score some time later. One may speculate that the allocation of very low scores (0-3) may be better recollected than intermediate scores, though there is little evidence for this.

It is rare for a resuscitator to consciously use the full Apgar score to determine how resuscitation should proceed, as originally intended. The combination of respiratory effort, colour, and heart rate guides most people, although colour may contribute little to the assessment. ${ }^{2}$ The final two items must then be estimated rather than assessed at the correct time. Even simultaneously recorded scores vary between observers: one case history based survey finding correct scoring in only $68 \%$ cases. $^{3}$

The Apgar score makes no allowance for the present day practice of intubation, not widely performed in the early 1950 s. How does one allocate a score for respiratory effort in a child breathing spontaneously but also receiving intermittent positive pressure ventilation? Can such a baby receive a score of 10 ? Our practice is to allow a maximum of 1 in this situation.

The application of the Apgar score to very preterm infants has also been questioned. Such infants frequently have low scores and may be assessed during active resuscitation or elective intubation. Reflex irritability, muscle tone, and respiratory effort may all be less pronounced in immature compared with mature infants. ${ }^{4}$ Preterm infants with low Apgar scores tend to have higher $\mathrm{pH}$ values than similarly depressed term infants. ${ }^{5}$ The five minute Apgar score is positively correlated with birth weight and is higher in small for gestational age infants compared with their appropriately grown counterparts. ${ }^{6}$

The problems associated with recording the Apgar score are thus easy to identify. Some can be overcome by better recording of data during resuscitation, perhaps using audio recording during resuscitation - an old idea ${ }^{7}$ - and accepting differing cut off points or standards for very preterm children. Identification of the contribution of component scores for preterm children is required. The glaring defect in the practical measurement of the score is, however, its inherent inaccuracy between observers, something that may not be easily overcome.

\section{Attempts to improve on Apgar's original score}

There have been few studies which have evaluated the relative contribution of the constituent parts of the score. There are complex relationships between the various components of the score. Producing a weighted score removes the simplicity of the original ${ }^{8}$ and there have been no successful attempts to replace the score with a viable alternative. In one elegant study the score allocated for colour was found to contribute little to the prediction of umbilical $\mathrm{pH}$, arterial carbon dioxide tension, and base excess, in contrast to the other items. ${ }^{2}$ The authors proposed that the score be used without colour, giving a maximum score of 8 . Such a score would have the advantage of reducing the error that may be introduced in the assessment of dark skinned infants. ${ }^{9}$ Perhaps this suggestion bears re-evaluation.

\section{Relationship with other measures of fetal} compromise

Much of the criticism that is levelled against the Apgar score is based upon the seemingly poor relationship that it has with other measures of perinatal compromise (fetal 
heart rate abnormalities, meconium staining of amniotic fluid, acidosis at birth) and the equally poor relationship it appears to now have with survival, neonatal hypoxicischaemic encephalopathy, or later neuromotor impairment. This criticism would have more weight if other measures of fetal compromise had any closer relationship with outcome.

Umbilical arterial or venous $\mathrm{pH}$ have been proposed as better markers than Apgar score, although there is little evidence that $\mathrm{pH}$ is any better. ${ }^{10} 11$ The relationship between Apgar score and umbilical $\mathrm{pH}$ is not clear. Sykes and colleagues demonstrated a poor correlation except at the extremes of acidosis, ${ }^{12}$ confirming an observation made as long ago as $1958 .{ }^{13}$ None the less, studies have continued to explore this relationship: in one recent study $61 \%$ of children with low one minute Apgar score $(<7)$ had a normal pH $(>7 \cdot 15) ; 74 \%$ of children with a low $\mathrm{pH}$ had a normal Apgar score. ${ }^{14}$ Umbilical $\mathrm{pH}$ may explain only as little as $8 \%$ of the variance in one minute Apgar score..$^{15}$ In a further study, by five minutes after birth only $11 \%$ of acidotic infants had low Apgar scores and only $41 \%$ of those with low five minute Apgar scores were acidotic. ${ }^{16}$

A closer relationship with $\mathrm{pH}$ has been proposed if colour is excluded ${ }^{2}$ and the Apgar score is measured immediately after birth, ${ }^{17}$ but each remains a poorly sensitive measure of the other. The correlation between Apgar score and umbilical $\mathrm{pH}$ may become reliable only in those severely depressed infants with very low $\mathrm{pH}$ values $(<7.00)$ and very low Apgar scores $(<3) .{ }^{15}{ }^{18}$ It has been proposed that those chronically asphyxiated infants with a corrected metabolic acidosis, who do not respond to further hypoxic stress with increasing acidosis, may be at greatest risk and that the ability to respond to hypoxia with acidosis is a good prognostic sign. ${ }^{11}$ It would appear therefore that acidosis and Apgar score are measures of different processes which at best appear to be only loosely related.

Abnormalities of the fetal heart rate pattern and the presence of meconium in the amniotic fluid are used to predict risk of asphyxia and to determine the management of labour. However, monitoring the fetus during labour may not improve the outcome. ${ }^{19}$ Neither fetal heart rate pattern nor the presence of meconium are good indicators of umbilical pH or Apgar score. In one study, although the besi correlate of $\mathrm{pH}$ was fetal heart rate pattern, it only explained $12 \%$ of the variance in $\mathrm{pH}$. Mode of delivery and the presence of meconium were the best correlates of Apgar score but even with the addition of fetal heart rate pattern only $17 \%$ of the variance was explained. ${ }^{15}$ Most of the variance in Apgar score, or $\mathrm{pH}$ for that matter, remains unexplained by abnormalities in the progress of the perinatal period as currently assessed.

Other markers of immediate neonatal condition, such as time to onset of spontaneous breathing/first breath or need for intubation suffer from the same disadvantages as the Apgar score and are likely to be less discriminatory, being single item measures and dependent upon maternal medication. They have not been systematically studied.

\section{Relationship of Apgar score with neonatal and later outcome}

The best clinical indicator that an infant has suffered significant asphyxial insult after birth is the neurological course over the days immediately after birth. The grade of postasphyxial encephalopathy (graded as mild, moderate, or severe ${ }^{20}$ ) is closely associated with a range of impairment ranging from early mortality, through severe impairment, to learning disabilities at school age. ${ }^{212}$ Few studies that attempt to study fetal condition or compromise assess the neonatal or later progress of the infants.
In the absence of the provision of good neonatal resuscitation and follow on care directed at limiting damage from asphyxia, the low Apgar score may identify infants with a high mortality and morbidity. ${ }^{22}$ In a recent study from Zimbabwe this would appear to still be the case: among 84 term newborns with five minute Apgar scores of 5 or less, 17 failed to establish respiration, 44 had moderate or severe hypoxic-ischaemic encephalopathy, and $42 \%$ died. $^{23}$

Most studies of fetal condition and outcome, however, come from centres with highly developed perinatal services, where the predictive value of the Apgar score is less clear. In one study Apgar score, cord $\mathrm{pH}$, and lactate all demonstrated poor sensitivity and positive predictive value for 1 year neurological outcome. ${ }^{16}$ Other studies have failed to document associations with Apgar score of assessments made up to 17 years after birth. ${ }^{24}$ The analysis of the antecedents of cerebral palsy from the National Collaborative Perinatal Project observed that a low Apgar score had to persist through to 20 minutes before an association could be found. ${ }^{25}$ In contrast, studies of neonatal mortality, asphyxial encephalopathy, or neurological impairment may identify a greater proportion of children with low Apgar scores among index groups, compared with controls. Since its first description the predictive effect of the Apgar score has been considerably weakened by the institution of prompt and effective neonatal care.

It is unlikely that the prediction of neonatal morbidity after perinatal asphyxia will be made by any single measurement. The use of blood lactate, creatine kinase, and other metabolic markers has not been attended with much success. One group has recently proposed a scoring system for neonatal organ dysfunction after perinatal asphyxia using a combination of the five minute Apgar score, an arterial base deficit measurement in the first postnatal hour, and the result of fetal monitoring. ${ }^{26}$ This composite score achieved a sensitivity of $94 \%$ and a specificity of $81 \%$, far better than any single measure. Further prospective studies are needed to confirm this. It would seem logical that a selection of markers combined will prove more predictive than any one alone.

\section{Should we still record the Apgar score?}

In a leading article in 1989, the Lancet called for the Apgar score to be 'pensioned off. ${ }^{27}$ There was no attempt to suggest a replacement, nor how one should record the state of the infant at birth. I suspect that much of the concern about the Apgar score in the literature results from incorrect interpretation of a low Apgar score as being synonymous with asphyxia, which it is not, and the use of this erroneous opinion in medicolegal work.

Although we should remain cautious in our interpretation of an individual Apgar score, it has value as a descriptor of the condition of the infant at birth. It is not by itself a useful outcome measure, nor does it predict the further progress of the infants reliably. We should maintain vigilance that it is measured as accurately as possible in each delivery suite and continue to search for other measures that may better indicate neonatal condition immediately after birth. Although imperfect, it would seem a little premature to pension the Apgar score off yet.

NEIL MARLOW

Department of Child Health,

Bristol Maternity Hospital,

Southwell Street,

Bristol BS2 8EG

1 Apgar V. A proposal for a new method of evaluation of the newborn infant. Current Research in Anesthetic Analgesia 1953;32:260-7. 
2 Crawford JS, Davies P, Pearson JF. Significance of the individual components of the Apgar Score. Br $\mathcal{F}$ Anaesth 1973;45:148-58.

3 Clark DA, Hakanson DO. The inaccuracy of Apgar scoring. $f$ Perinatol 1988;8:203-5.

4 Catlin EA, Carpenter MW, Brann-BS, et al. The Apgar score revisited: influence of gestational age. $\mathcal{F}$ Pediatr 1986;109:865-8.

5 Goldenberg RL, Huddleston JF, Nelson KG. Apgar scores and umbilical pH in preterm newborn infants. Am $\mathcal{F}$ Obstet Gynecol 1984;149:651-4.

6 Stark CF, Gibbs RS, Freedman WL. Comparison of umbilical artery pH and 5-minute Apgar score in the low-birth-weight and very-low-birthand 5-minute Apgar score in the low-birth-weight

7 Davies PA, Robinson RJ, Scopes JW, Tizard JPM, Wigglesworth JS. Medical care of newborn babies. Clinics in Developmental Medicine No Medical care of newborn babies. Clinics in Developmental Medicine

8 Apgar V, Holoday DA, James LS, Wesibrot IM. Evaluation of the newborn infant - a second report. FAMA 1958;168:1985-8.

9 Yama AZ, Marx GF. Race and Apgar scores (letter). Anaesthesia 1991;46: $330-1$

10 Johnsten BE, Johnson TRB, Nelson JP. Umbilical cord pH and Apgar score as an index of neonatal health. Am F Obstet Gynecol 1987;157: 843-8.

11 Dennis J, Johnson A, Mutch L, Yudkin P, Johnson P. Acid-base status at birth and neurodevelopmental outcome at four and one-half years. $\mathrm{Am} \mathcal{F}$ Obstet Gynecol 1989;161:213-20.

12 Sykes GS, Molloy PM, Johnson P, et al. Do Apgar scores indicate asphyxia? Lancet 1982;i:494-6.

13 James LS, Weisbrot IM, Prince CE, Holaday DA, Apgar V. The acid-base status of human infants in relation to birth asphyxia and the onset of respiration. $\mathcal{F}$ Pediatr 1958;52:379-94

14 Hoffmann AL, Hjortdal JO, Secher NJ, Weile B. The relationship between Apgar score, umbilical artery $\mathrm{pH}$ and operative delivery for fetal distress in 2778 infants born at term. Eur $\mathcal{F}$ Obstet Gynecol Reprod Bio 1991;38: in 2778 . 101 .
15 Steer PJ, Eigbe F, Lissauer TJ, Beard RW. Inter-relationships among cardiotocograms in labour, meconium staining of the amniotic fluid, arterial cord blood pH and Apgar scores. Obstet Gynecol 1989;74:715-20.

16 Ruth VJ, Raivio KO. Perinatal brain damage; predictive value of metabolic acidosis and the Apgar score. BMF 1988;297:24-7.

17 Marx GF, Mahajan S, Miclat MN. Correlation of biochemical data with Apgar scores at birth and at one minute. Brf Anaesth 1977;49:831-3.

18 Gilstrap LC, Leveno KJ, Burris J, Williams ML, Little BB. Diagnosis of birth asphyxia on the basis of fetal $\mathrm{pH}$, Apgar score and newborn cerebral dysfunction. Am 7 Obstet Gynecol 1989;161:825-30.

19 MacDonald D, Grant A, Sheridan-Pereira M, Boylan P, Chalmers I. The Dublin randomized controlled trial of intrapartum fetal heart rate moniDublin randomized controlled trial of intrapart

20 Sarnat HB, Sarnat MS. Neonatal encephalopathy following fetal distress. Arch Neurol 1976;33:696-705.

21 Robertson CMT, Finer NN, Grace MGA. School performance of survivors of neonatal encephalopathy associated with birth asphyxia at term. f Pediatr 1989;114:753-60.

22 Apgar V, James LS. Further observations on the newborn scoring system. Am $\mathcal{F}$ Dis Child 1962;104:419-28.

23 Nathoo KJ, Chimbra TH, Mtiavalye LA. Mortality and immediate morbidity in term babies with low Apgar scores. Ann Trop Paediatr 1990;10: 239-44.

24 Seidman DS, Paz I, Laor A, Gale R, Stevenson DK, Danon YL. Apgar scores and cognitive performance at 17 years of age. Obstet Gynecol 1991; 77:875-8.

25 Nelson KB, Ellenberg JH. Antecedents of cerebral palsy. Multivariate analysis of risk.N Engl भुMed 1986;315:81-6.

26 Portman RJ, Carter BS, Gaylord MS, Murphy MG, Thieme RE, Merenstein GB. Predicting neonatal morbidity after perinatal asphyxia: a scoring system. Am 7 Obstet Gynecol 1990;162:174-82.

27 Anonymous. Is the Apgar score outmoded? Lancet 1989;i:591-2.

\section{Metabolic acidosis in newborn infants}

The maintenance of a proton (hydrogen ion) concentration within a relatively narrow range is essential for normal cellular function. This is as true for newborn infants as it is for older children and adults. Although very large quantities of protons are produced during normal metabolism by the oxidation of substrates to carbon dioxide, hydrolysis of adenosine triphosphate and reduction of adenosine dinucleotides, these are effectively removed by associated reactions. In health net production of protons results primarily from the catabolism of sulphur-containing amino acids and hydrolysis of nucleic acids in the diet. In order to maintain a normal $\mathrm{pH}$, hydrogen ions are buffered by extracellular and intracellular proteins, inorganic phosphate, and bicarbonate. Net loss of protons from the body results from loss of carbon dioxide in expired gas (with hydrogen ions being effectively lost to water) and from the excretion of dihydrogenphosphate and ammonium ions by the kidney. Reabsorption of sodium bicarbonate by renal tubules, although not resulting in any net loss of protons, is also important in maintaining normal acid-base balance.

Respiratory acidosis in the newborn period may be due to disorders such as respiratory distress syndrome, transient tachypnoea of the newborn, and meconium aspiration, and results from the reduction in carbon dioxide excretion by the lungs with a subsequent increase of carbonic acid. Metabolic acidosis, defined as the accumulation of non-carbonic acid equivalents, arises from excessive production or inadequate excretion of hydrogen ions or from an increased loss of bicarbonate. In practice metabolic acidosis may result from birth asphyxia, cold stress, hypovolaemia, sepsis, congenital heart disease (particularly hypoplastic left heart syndrome, coarctation and interruption of the aortic arch), renal disease (for example polycystic kidneys, obstructive nephropathies, renal tubular acidosis), maternal acidosis, and inborn errors of metabolism. ${ }^{1}$ Acidosis has also been reported in newborn infants with imperforate anus and rectovaginal fistula, ${ }^{2}{ }^{3}$ neonatal diabetes, ${ }^{4}$ benzyl alcohol poisoning, ${ }^{5}$ saline flushes, ${ }^{6}$ parenteral nutrition, ${ }^{7}$ and in those fed with casein formulas $^{8}$ or with goats' milk. ${ }^{9}$

Although the mechanisms by which acidosis causes harm are not fully understood, severe acidosis is associated with disturbances in cerebral blood flow, periventricular haemorrhage, leucomalacia, increased peripheral vascular resistance, and decreased myocardial function. ${ }^{10}{ }^{11} \mathrm{~A}$ fall in cardiac output and poor tissue perfusion may increase tissue hypoxia and lead to worsening acidosis.

Preterm infants are more susceptible to many disorders that cause metabolic acidosis such as cold stress, infection, and respiratory distress syndrome and have a reduced capacity to prevent and correct acidosis. Although the kidneys will respond to an acid load in a qualitatively similar manner to term infants, immaturity of renal function results in a reduced glomerular filtration rate, lower tubular bicarbonate threshold, and increased urinary sodium loss. ${ }^{12}$ Additionally any concomitant respiratory disease may restrict the ability of the preterm infant to compensate for a metabolic acidosis by increasing the excretion of carbon dioxide.

\section{Inborn errors of metabolism}

Although individually uncommon, inborn errors of metabolism are not a rare cause of metabolic acidosis in the newborn and are more likely where there is consanguinity or a family history of unexplained neonatal deaths or illness. Their presentation is, however, usually non-specific, and unless appropriate investigations are undertaken the correct diagnosis may be missed. Characteristically, but not exclusively, infants with an inborn error of metabolism are born at term and are initially well. In preterm infants acidosis is much more likely to be secondary to other causes such as septicaemia but the possibility of an inherited metabolic disorder should still be borne in mind. The main groups of inborn errors that may present with a severe metabolic acidosis are (i) defects of pyruvate metabolism and the mitchondrial electron transport chain, (ii) the organic acidaemias, and (iii) defects of gluconeogenesis.

\section{Investigations}

In the majority of sick infants a full infection screen and liver function tests will usually be undertaken and concentrations of arterial blood gases, blood glucose, and 